\title{
Evaluating the Real-World Performance of 5G Fixed Wireless Broadband in Rural Areas
}

\author{
Raouf Abozariba, Eleanor Davies, Matthew Broadbent and Nicholas Race \\ School of Computing and Communications, \\ Lancaster University, Lancaster, LA1 4WA, UK \\ \{r.abozariba, eleanor.davies, m.broadbent, n.race\}@lancaster.ac.uk
}

\begin{abstract}
Rural communities are often neglected in the rollout of communication infrastructure, delaying or even preventing the delivery of common digital services that are now ubiquitous in modern society. Addressing this technological gap is of paramount importance. In this paper, we analyze the performance of two new and emerging $5 G$ networking technologies, each capable of overcoming some of the challenges present in rural environments. Our analysis is based around a series of deployments of TV White Space (TVWS) and millimetre wave (mmWave) technologies across rural parts of the UK. These deployments are enhanced with a custom designed monitoring platform that includes adaptive streaming video clients that enable the accurate assessment of end-user quality of experience. Our findings demonstrate that TVWS solutions, which operate on an unlicensed TV band, have clear potential for providing stable broadband services in those areas. Our results also show that although mmWave-based solutions can provide sub-gigabit-speed, they are vulnerable to excessive rainfall and are limited to short line-of-sight usage, making them less applicable to deployment in irregular terrain.
\end{abstract}

Index Terms-mmWave, TVWS, Wireless Broadband.

\section{INTRODUCTION}

For thirty years the Internet has achieved a remarkable transformation in how we live, communicate and access information. It is estimated that half of the worlds population (nearly 3.9 billion people) is now online [1]. However, in many parts of the globe, and even in the developed world, there remain large pockets where the Internet is unavailable or available with limited service quality, making many applications and services inaccessible. This is largely the result of the failure of the previous generation of cellular networks such as $3 \mathrm{G}$ and $4 \mathrm{G}$ to reach rural areas, combined with a lack of incentives from major Internet Service Providers (ISPs) to roll out wired broadband coverage such as VDSL and fibre, which requires significant expenditure on infrastructure. As a consequence, various alternative solutions are emerging, including those powered by TV White Space and millimetreWave (mm-Wave) spectrum for rural broadband expansion. These solutions are considered economical in many parts of the world but detailed (and up-to-date) information relating to real world deployments in rural areas is far more limited. Hence, it is vital to analyze the effectiveness of such solutions in their ability to provide key Internet services to a broad range of users.
TVWS has greatly improved the connectivity to many rural communities around the world. There are many TVWS deployment in many parts of the world under different topologies (including $\mathrm{P} 2 \mathrm{P}$ and $\mathrm{P} 2 \mathrm{MP}$ ), resulting in new intelligence about TVWS spectrum capabilities [2]-[4]. These efforts, however, lack the support of continuous measurement which is essential for capturing time-varying changes in the networks and do not assess geographic diversity in which such solutions are typically deployed [5]-[10]. In addition, the radios used in those deployments are capable of communicating through only one channel $(6 / 8 \mathrm{MHz})$ per connection. There are now many solutions which support channel aggregations of up to three channels. Work on investigating such solutions is limited.

Early work evaluating mmWave capabilities has focused mainly on propagation modelling [11]-[13]. The work presented in [11] investigated the feasibility of using $60 \mathrm{GHz}$ in the picocell range by characterizing attenuation, sensitivity to movement and blockage, and interference in urban settings. The measurements are centred around Transmission Control Protocol (TCP) throughput, which is obtained considering distances up to 200 meters. Although the literature in this domain provides detailed analyses of the mmWave spectrum characteristics, it does not fully capture the Quality of Experience (QoE) perceived by the end user [14], [15].

This paper analyses the real-world performance of TVWS and mmWave technologies through a series of $5 \mathrm{G}$ trials within rural parts of the UK. These findings are based on a customdeveloped monitoring platform, that enables us to obtain an accurate picture of the end-user QoE.

The rest of the paper is organized as follows. Section II presents the current TVWS and mmWave solutions and methods of deployments. In Section III the measurement framework is described followed by the results in Section IV. The challenges of the TVWS and mmWave technologies are presented in Section V and Section VI respectively. Section VII discusses the future upgrades and longevity of the technologies. In Section VIII we conclude with a few remarks.

\section{FIXED WIRELESS BROADBAND SOLUTIONS}

In this section we categorize two technologies used to deliver fixed wireless broadband in rural settings: 


\section{A. TV White Space (TVWS)}

TVWS technology is now in its commercial phase and there are manufacturers and developers specializing in providing hardware to support long-range connectivity, which is appropriate for rural settings. TVWS takes advantage of advancements made in cognitive radio technology, such as dynamic spectrum allocation, enabled through the use of a TVWS database (TVWSDB), designed to support same-band access by multiple concurrent users. It is also a license-exempt spectrum in many countries.

In this study, we investigate five TVWS testbeds in areas categorized as rural. Each testbed consists of a number of Customer Premises Equipment (CPEs) connected via Ultra High Frequency (UHF) radio to their respective base station (BTS). The CPEs are located at varying distances, within the azimuth plane of the BTSs antenna. The testbeds are spread across a geographically diverse area within the UK, incorporating England, Scotland and Wales.

\section{B. millimetre-Wave (mmWave)}

mmWave spectrum represents the core spectrum for enabling 5G cellular networks and many P2P backhaul solutions. It also enables the delivery of multi-gigabit Internet broadband connectivity wirelessly in rural areas. The crucial feature of unlicensed $60 \mathrm{GHz}$ mmWave used in this solution is that the available spectrum is $6.8 \mathrm{GHz}$, which is 20 times more than that in the TVWS counterpart. There are multiple vendors, who are developing mmWave solutions, which can deliver super-fast Internet access, by taking advantage of beamforming technology and license-exempt spectrum bandwidths in the $60 \mathrm{GHz}$ range.

To test the effectiveness of this technology in the rural environment, we built a testbed consisting of a BTS connected to six CPEs through mmWave channels, distributed within the azimuth plane of the BTSs antenna (about 90 degrees angle) with the nearest CPE located at 364 metres and the furthest at 693 metres. The kit is also equipped to support transmissions over $5 \mathrm{GHz}$ spectrum, which is used as a backup spectrum when weather adversaries such as heavy rainfall are encountered. This makes the radio equipment more resilient to interference from weather conditions.

\section{Measurement Framework}

To monitor the networks described above, we have developed our own monitoring framework. The monitoring framework is divided into three distinct layers; the application layer, network layer and physical layer. The application layer and network layer generate statistics collected using a number of monitoring devices we place within the network, whereas the physical layer obtains data via monitoring protocols built into the network infrastructure itself.

\section{A. Physical Layer}

The physical layer principally uses the Simple Network Management Protocol (SNMP) to perform data collection and display of key statistics. In the event where support of SNMP is not available, we have developed an API to communicate with the BTS via JSON. The API spoofs requests made by the WebUI of the BTS to obtain a range of information such as the current channel usage, the temperature the transmitter power, channel bandwidth utilization, modulation scheme and received signal strength indicator (RSSI)/signal to noise ratio (SNR). These are common statistics, which network operators often monitor to determine the health of the network, but may not necessarily correlate with the QoE the user is actually encountering. These statistics are collected once every minute.

\section{B. Network Layer}

This is a set of active measurements, which perform endto-end measurements to determine facets including jitter, latency and throughput. These measurements are made between the customer LAN and a remote server to determine the performance between the devices, and provide data on the quality of the service. The active monitoring device is capable of reporting the current bandwidth used by a given client, and allow appropriate actions to be performed. Importantly, the performance observed are real-world measurements, they include the overhead introduced with TCP and indeed the wider network infrastructure and are therefore reflective of the real world performance.

The latency of the system focuses on the round trip time that it takes for packets to traverse the network to a remote server using ICMP echo requests. Jitter is an extension on latency measurements, observing the variation of latency over time. Packet delay variation indicates how the latency varies which has an impact on the quality of the provided service.

The throughput of the system is quantified as the bits per second that can traverse the network after accounting for buffer and processing overheads - the bandwidth available for the user to utilize. The throughput will be affected by factors such as congestion, time and arbitrary errors and may differ significantly to the sampled bandwidth of the link from physical layer monitoring.

\section{Application Layer}

The application layer focuses on the QoE observed at the user-centric level of the network, detailing the real-world benefits of the system as perceived by real-world users. In order to measure the QoE, our monitoring device streams a 10-minute DASH video once an hour and examines the experience perceived by the user. This measuring approach comes at the expense of average bandwidth consumption of around 2.6 Mbps with the peak bandwidth consumption of 8 Mbps.

We analyze events which are detrimental to QoE such as stalling, buffering and resolution. This, combined with observing how long the playback remained within each resolution, gives insight into whether or not the network is meeting the performance stated in the network and physical layers. Stalling and buffering are both undesirable from a user experience standpoint and lead to a great deal of annoyance, but are necessary in the case of slow and unstable networks. For 
reasons of brevity, we only report the video quality from the resolution perspective.

\section{TABLE I}

\begin{tabular}{llcc}
\hline Location & Technology & Bandwidth & Backhaul \\
\hline Lancaster & VDSL & $25 \mathrm{MHz}$ & N/A \\
Auckley & mmWave & $2000 \mathrm{MHz}$ & 1Gbps fiber \\
Kintyre Cottages & TVWS & $2 \times 8 \mathrm{MHz}$ & 30Mbps VDSL \\
Inverness & TVWS & $2 \times 8 \mathrm{MHz}$ & $<15 \mathrm{Mbps} 5 \mathrm{Ghz}$ \\
Bardney & TVWS & $2 \times 8 \mathrm{MHz}$ & $1 \mathrm{Gbps}$ fiber \\
Ninebanks Youth Hostel & TVWS & $2 \times 8 \mathrm{MHz}$ & 1Gbps fiber \\
Nenthead Mines & TVWS & $2 \times 8 \mathrm{MHz}$ & 1Gbps fiber \\
\hline
\end{tabular}

\section{RESULTS}

In this section, we provide detailed analyses to characterize the performance of state-of-the-art TVWS and mmWave technologies deployed by two Wireless Internet Service Providers (WISPs): QuickLine Communications and Broadway Partners. In table I, Broadway maintains the Kintyre Cottages and Inverness (both in Scotland) installations and QuickLine operate the Auckley, Bardney, Ninebanks Youth Hostel and Nenthead Mines installations. It is important to note that the vendors used by the companies for TVWS differ with Broadway utilizing hardware supplied by HuWoMobility and QuickLine deploying 6Harmonics hardware. The $60 \mathrm{GHz}$ hardware deployed by QuickLine in Auckley is manufactured by IgniteNet and has the capability to operate at multiple Gbps.

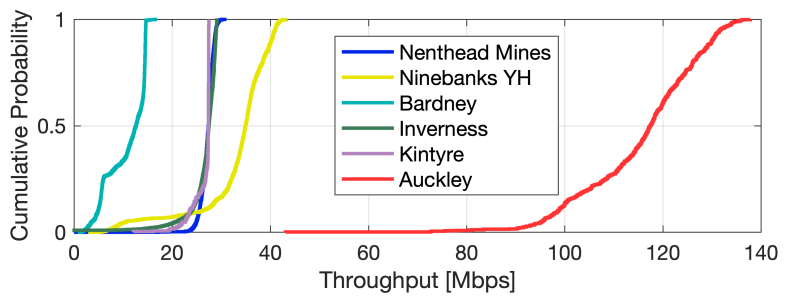

Fig. 1. ECDF of the download throughput from monitoring probes to target public servers.

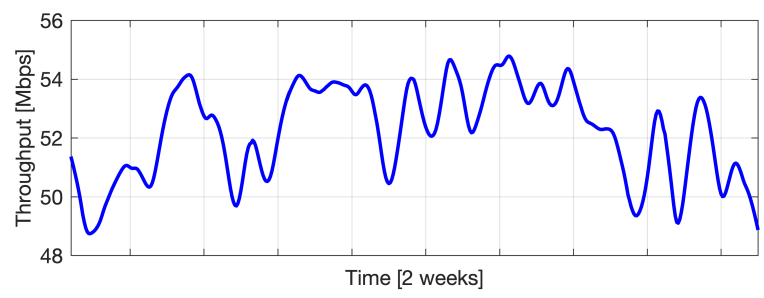

Fig. 2. Throughput recorded over two weeks between the BTS and CPE in Inverness using iperf test, achieved through deploying two monitoring devices on either end of the link. The mean is $52.14 \mathrm{Mbps}$ and the standard deviation is 1.6. Download and upload capacities are symmetric given the 1:1 ratio transmission across the entire channel.

\section{A. Throughput}

Figure 1 presents the empirical cumulative distribution function (ECDF) of the measured download speed at five different locations using TVWS radio and at Auckley using $60 \mathrm{GHz}$ mmWave Radio. Measurements were taken over a 7day period, in 5 minutes intervals. From the figure, it is evident that the TVWS technology operating over two $8 \mathrm{MHz}$ bonded channels can support internet speed between 20-30 Mbps on an asymmetric connection. The distance between the BTSs and the respective CPEs at each location are in the range of 6-10 km. This suggests that with a good backhaul connection the TVWS is a promising candidate, which potentially could substitute traditional wired broadband solutions. What is more noteworthy among these findings is the stability of the service, where over the entire monitoring period, no outages were observed. Furthermore, despite the diversity of locations in which these wireless networks are deployed and the variety of backhaul capabilities, the performances are relatively homogeneous.

Using passive monitoring, we capture the RSSI data from the network in Bardney. As shown in Figure 3, throughout the monitoring period ( 5 days), the signal strength fluctuate between $-75 \mathrm{dBm}$ and $-92 \mathrm{dBm}$. The drop of the signal strength occurs mostly between 6 am and 6 pm every day, which suggest there is interference from another source in the region operating between these hours. The observed interference has a negative impact on the throughput, as shown in Figure 1.

Another monitoring device is installed to monitor the QoS provided by mmWave radio. The speed achieved using mmWave (see Figure 1-Auckley) radio is 4-5 times faster than its TVWS counterpart. mmWave radios are known to provide high capacity and high-speed data transmission but require good backhaul connection in order to support multiple CPEs with the same level of service.

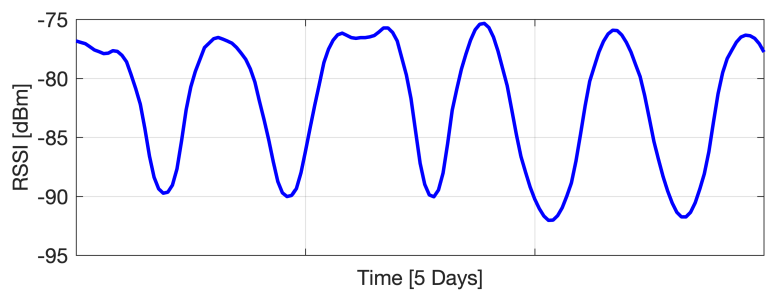

Fig. 3. The Received Signal Strength Indicator (RSSI) status of the radio frequency (RF) power input to the transceiver of CPE connected through TVWS channels at Bardney testbed.

\section{B. Latency}

Figure 4 presents the latency measured at four different testbeds operating in TVWS radio. Two metrics are considered: round-trip time (RTT) and jitter, also known as variance in the latency. Latency below $40 \mathrm{~ms}$ as observed in all testbeds over 7-day monitoring period is considered acceptable by the major traffic categories such as video, voice and data. The recorded jitter values below $25 \mathrm{~ms}$ are also considered satisfactory by most applications.

To evaluate the performance of the TVWS link between the user and the base station, we attached one monitoring probe to the base station and another to a user's router in the testbed 
in Inverness. This has allowed for more rigorous tests on the TVWS wireless link itself. Figure 4 (bottom-right) shows that the TVWS link achieves low average latency and low jitter below $5 \mathrm{~ms}$. The same measurements were performed on the mmWave radio testbed in Auckley. The average RTT was $12.66 \mathrm{~ms}$ and jitter was $18.19 \mathrm{~ms}$.

\section{Video Quality}

Despite the fact that Internet video streaming has grown tremendously over the last decade, it is still considered one of the most challenging applications in modern networks [16]. Video streaming requires the steady flow of information and delivery of packets in a timely manner. Without this, a smooth viewing experience cannot be provided to the end user. Wireless radio networks have difficulties in providing such a service in noisy environments, particularly when the spectrum is shared. Therefore, our focus has been on the video quality monitoring. Video quality is the primary metric used to determine QoE and, as demonstrated by these results, can provide excellent insights into the real world performance of the network from a user-centric standpoint. The key metric used is the average time spent in each resolution for each testbed. All networks will experience some time in lower resolutions as the stream initializes, with faster networks providing greater bandwidth, allowing the stream to jump to higher resolutions faster when compared to slower networks. Network stability also plays a significant role as packet loss due to transient RF events, can cause the TCP congestion response to be triggered forcing DASH to reduce the resolution to attempt to continue with smooth playback.

Figure 5 demonstrates the differences between the various technologies used in the project. Lancaster is provided as a benchmark and is a typical high-performance VDSL connection, common in most urban and semi-urban areas in the UK. The Inverness installation performs notably badly. This is because the non-5G backhaul for the network has an inherent high latency $(>50 \mathrm{~ms})$ and low throughput $(<15 \mathrm{Mbps})$ service provision. The TCP throughput of the TVWS segment of this installation has been observed to be $>50 \mathrm{Mbps}$, as seen in figure 2. Bardney is a $10 \mathrm{~km}$ TVWS link in Lincolnshire, where the spectrum is fairly congested. As a result, the throughput is often severely limited and lossy. This can be clearly seen in figure 5 where, on average, the playback is only achieving full HD approximately $10 \%$ of the time.

The same technology as used in Bardney has was deployed to Ninebanks Youth Hostel and Nenthead mines in rural Cumbria. Here the interference from primary and secondary operators is lower, leading to an improved performance, with the system being able to stream in HD for $>80 \%$ of the time. The backhaul for this location is a $400 \mathrm{Mbps}$ microwave link to a $1 \mathrm{Gbps}$ fibre backhaul to Manchester.

Kintyre utilizes TVWS, however, uses VDSL limited to $30 \mathrm{Mbps}$ as the backhaul. Since the installation is in a very rural area, the interference is minimal allowing for the full bandwidth of the TVWS link to be used, although it is unclear what that performance is due to physical layer monitoring being unavailable. The observed performance is good, with HD streaming available in excess of $80 \%$ of the time.

Auckley uses $60 \mathrm{GHz}$ technology and outperforms our VDSL benchmark with HD streaming being available for more than $95 \%$ of the time. This technology is used in a densely populated urban housing estate, which is in a rural area, and has no fibre or VDSL connectivity. The testbed has six homes connected, all with users reported performance stating the quality of service is excellent, as evidenced by our monitoring. These results correlate with Figure 1 with the exception of Inverness, which only measures throughput between the BTS and the CPE.

\section{Challenges of TVWS technology}

At its current format, the TVWS technology offers high Internet speed (around $30 \mathrm{Mbps}$ ) with low latency (30 ms) and has the potential to support multiple use cases. However, there are a number of challenges, which limit the technology from addressing the connectivity of rural regions as expected. We summarize some of these challenges below:

Limited channel availability: A key commodity to transmit data via an electromagnetic signal is the size of the channel bandwidth. The broader the bandwidth, the more data can be transmitted. However, the availability of spectrum bandwidth with characteristics suitable for a rural environment such as the TVWS spectrum is limited. In addition, it is reported that the $700 \mathrm{MHz}$ spectrum $(694-790 \mathrm{MHz}$ ) will be auctioned by Ofcom, the UK spectrum regulator, for mobile broadband usage (4G LTE/5G $700 \mathrm{MHz}$ ), which means a large chunk of the band will become unavailable to white space devices.

High noise-floor in the TVWS spectrum limits the channel selection: Due to the geographical landscape of the UK and the number of transmitters within the TVWS, and add to it the propagation of the signal within this range of the spectrum, the interference level is high in many locations in the UK, making the number of channels available very limited.

Requires contiguous channel bonding to provide speeds above 30 Mbps: Increasing the speed beyond $30 \mathrm{Mbps}$ requires aggregating channels of three or more. The TVWS equipment, which represents the state-of-the-art technology used in testbeds, supports the bonding of up to 3 channels if the channels are contiguous. Aggregating channels is common in many wireless standards but not easy to achieve in TVWS, since it is a shared spectrum and users are allowed to transmit over the spectrum on overlay basis (or opportunistically), making channels sparse in this band. Given the limited number of channels in the spectrum, access to 3 contiguous channels has proven to be a rare occurrence.

Despite the shortcomings of the technology, outlined above, the performances show that, in situations where TVWS can be successfully deployed, it is an effective but interim solution. However, it is envisaged that as the technology matures, it will side further widespread adoption.

\section{Challenges of mmWave technology}

Despite the success achieved through mmWave technology, showcasing its potential to provide unprecedented speed in 

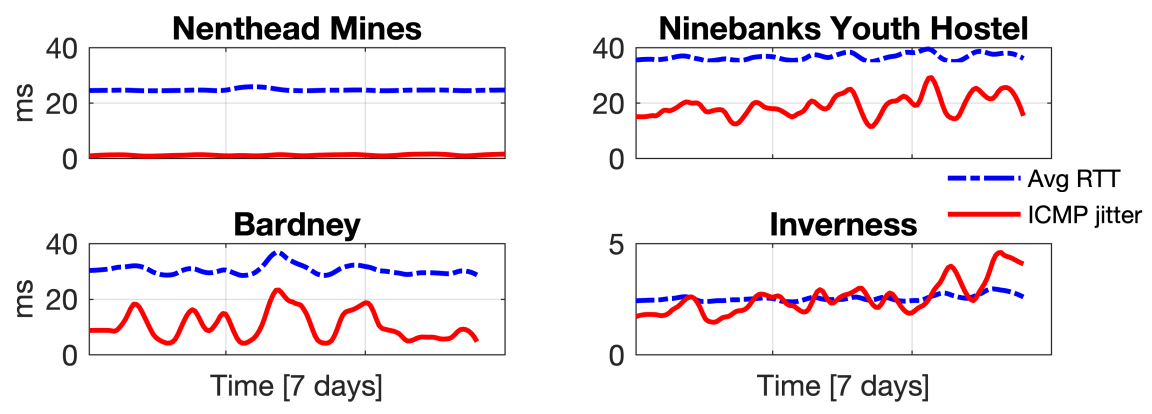

Fig. 4. Measured RTT (latency of the network) and jitter (varience of the network latency) from selected TVWS users to a remote server. The bottom right figure presents the latency of TVWS wireless link between base station and user.

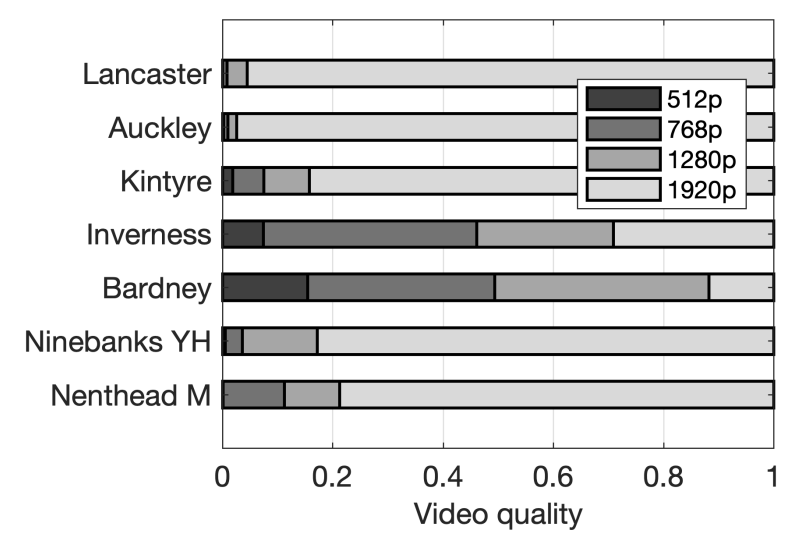

Fig. 5. Comparison between various broadband installations, from a QoE perspective. The graph reports the performance of the monitored users in terms of received resolution quality after 168 videos downloads at each point.

rural areas ( $>100 \mathrm{Mbps})$, it suffers from a number of drawbacks, which are outlined below:

Transmission range is below $1.5 \mathrm{Km}$ : The attenuation of signals in the mmWave band has low range, particularly under low power budget (Maximum permitted transmission power in the $60 \mathrm{GHz}$ unlicensed spectrum under Ofcom regulations is $10 \mathrm{dBm})$.

Dependency on Gbps backhaul availability: Due to the short signal transmission range in the $60 \mathrm{GHz}$ spectrum, to increase the coverage, the requirement of backhaul access points increase which, in turn, add to the complexity of the network design. This, of course, will raise the overhead cost of the network operators and would adversely reflect on the consumer bill.

Pairing transceivers require line-of-sight (LOS): Due to the high path loss of the signal between the transmitter and receiver through buildings, foliage and adverse weather, etc. in this frequency range signal transmission under Non LOS (NLOS) is limited (e.g., 55.3\% more path loss in NLoS compared to LOS propagation in rural environment). Increasing the height of the antenna at the BTS and CPE would improve the probability of establishing LOS path, however, due to customers home structure and tight planning permission constraints in some areas, this is not always possible.

Regulations on the usage of $60 \mathrm{GHz}$ band is limited to Fixed Wireless Systems: According to the latest Ofcom release in respect to $60 \mathrm{GHz}$ spectrum, usage of this band is only permitted for fixed wireless systems. This has implications on addressing agricultural use cases, such as precision farming, where mobile radio equipment is required to transmit video data collected by UAVs to a ground station, and to do this in real time.

Many of these challenges are currently being investigated by the research community and industry, and if successful, could vastly improve the connectivity in rural areas in the future.

\section{FUTURE UPGRADES AND LONGEVITY}

This section is to discuss possible future upgrades with respect to TVWS and mmWave based on current developments aimed at enhancing connectivity in rural areas. The data rate provided by the TVWS technology can be increased by combining multiple contiguous channels, subject to availability of bandwidth in a given location. Currently, TVWSDB spectrum channel scan shows the availability of two contiguous channels in some areas, while the availability of three contiguous channels is extremely limited. As the technology advances to enabling non-contiguous channel bonding (or aggregating individual channels together to form one broader channel to enable higher throughput and stability to the link), the possibility to achieve two, three or more channel bondings will be higher, providing further stability and reliability to the end user.

In addition, the emerging concepts of Network Function Virtualization (NFV) and Software-Defined Networking (SDN) will likely play a pivotal role in accelerating the development of TVWS technology. NFV and SDN will also enable services such as a content delivery network (CDN), another key technology contributing to improvement of accessibility and connectivity. Furthermore, Software Defined Radio (SDR) is expected to address challenges related to the process of filtering of unwanted signals at the RF receiver more efficiently by adapting to different environments without the need for expensive, impractical hardware solutions.

The fact that TVWSDB is the first real implementation of spectrum sharing by Ofcom using a database approach and 
yet to be tested in large scale deployment, suggest that future frameworks could be improved and more effective over time. Current TVWSDB is developed and maintained using signal propagation models and is considered to be stringent to limit interference and protect primary user transmission. As these propagation models are developed and become less strict and more accurate, better channel availability could be expected.

Available mmWave solutions, which is at the heart of many proposals for $5 \mathrm{G}$, are limited to a few hundred meters transmission ranges due to power restriction to limit interference and to allow spectrum to be coordinated by the users themselves rather than by regulators. However, a New York University Wireless research group led by T.S. Rappaport carried out experiments using guided directional beam in the $73 \mathrm{GHz}$ range in a rural area of Virginia, USA, achieving up to 10 $\mathrm{Km}$ transmission [17]. The success of mmWave transmission in rural areas over long distances promises higher broadband speed and undoubtedly will transform the connectivity experience in those rural areas. In addition, solutions such as mesh networks can be utilized to improve the spread coverage of mmWave under low power restrictions. For example, the work proposed in [18], which utilizes a distributed antenna system can be pursued.

Usage of $60 \mathrm{GHz}$ band is currently limited to Fixed Wireless Systems (FWS) according to the latest Ofcom consultation found in (Wireless Telegraphy Regulations, 2018). This has an implication on the support of use cases such as precision farming, which require equipment such as drones to be connected wirelessly to the internet to enable transmission of footage under beyond visual line of sight (BVLOS). However, as the mmWave technology matures, it is possible that the regulations will change in favour of a mobile wireless system. This will not only unlock precision farming use cases and virtual reality applications, but others as well, such as V2X communications (Vehicle-to-Vehicle or Vehicle-to-Infrastructure), which are paramount for the success of autonomous driving.

\section{CONCLUSION}

In this paper, we have implemented a monitoring framework which leverages a set of measurement tools to provide an in-depth view of real-world network performance. We have deployed this in a diverse set of rural testbeds across the UK, each using state-of-the-art TV White Space (TVWS) and millimetre wave (mmWave) technologies. The monitoring captures crucial metrics such as throughput, latency and video resolution quality over substantial periods of time. We also discussed the challenges of the technologies when deployed in real-world environments. In the future, we aim to deploy the monitoring framework alongside other technologies designed for rural areas, as well as increasing the number and size of deployments.

\section{ACKnowledgment}

This work was funded by the Department for Digital, Culture, Media \& Sport (DCMS) as part of the 5G Rural
Integrated Testbed (5GRIT) project under its 5G Testbeds and Trials Program.

\section{REFERENCES}

[1] J. Gozalvez, "5G worldwide developments [mobile radio]," IEEE Vehicular Technology Magazine, vol. 12, no. 1, pp. 4-11, 2017.

[2] R. Chandra, T. Moscibroda, P. Bahl, R. Murty, G. Nychis, and X. Wang, "A campus-wide testbed over the TV white spaces," ACM SIGMOBILE Mobile Computing and Communications Review, vol. 15, no. 3, pp. 2-9, 2011.

[3] P. Bahl, R. Chandra, T. Moscibroda, R. Murty, and M. Welsh, "White space networking with Wi-Fi like connectivity," ACM SIGCOMM Computer Communication Review, vol. 39, no. 4, pp. 27-38, 2009.

[4] A. Achtzehn, L. Simić, M. Petrova, and P. Mähönen, "Feasibility of secondary networks: Analysis methodology and quantitative study of cellular and Wi-Fi-like TVWS deployments," IEEE Transactions on Mobile Computing, vol. 14, no. 2, pp. 373-386, 2015.

[5] M. Masonta, L. Kola, A. Lysko, L. Pieterse, and M. Velempini, "Network performance analysis of the Limpopo TV white space (TVWS) trial network," in AFRICON 2015. IEEE, 2015, pp. 1-5.

[6] A. A. Lysko, M. T. Masonta, M. R. Mofolo, L. Mfupe, L. Montsi, D. L. Johnson, F. Mekuria, D. W. Ngwenya, N. S. Ntlatlapa, A. Hart et al., "First large TV white spaces trial in South Africa: A brief overview," in 2014 6th International Congress on Ultra Modern Telecommunications and Control Systems and Workshops (ICUMT). IEEE, 2014, pp. 407414.

[7] K. Ndlovu, Z. A. Mbero, C. L. Kovarik, and A. Patel, "Network performance analysis of the television white space (TVWS) connectivity for telemedicine: A case for Botswana," in 2017 IEEE AFRICON. IEEE, 2017, pp. 542-547.

[8] K. Ishizu, K. Hasegawa, K. Mizutani, H. Sawada, K. Yanagisawa, T. Keat-Beng, T. Matsumura, S. Sasaki, M. Asano, H. Murakami et al., "Field experiment of long-distance broadband communications in TV white space using IEEE 802.22 and IEEE 802.11af," in 2014 International Symposium on Wireless Personal Multimedia Communications (WPMC). IEEE, 2014, pp. 468-473.

[9] C. McGuire, M. R. Brew, F. Darbari, S. Weiss, and R. W. Stewart, "Enabling rural broadband via TV white space," in 2012 5th International Symposium on Communications, Control and Signal Processing. IEEE, 2012, pp. 1-4.

[10] S. Roberts, P. Garnett, and R. Chandra, "Connecting Africa using the TV white spaces: From research to real world deployments," in The 21st IEEE International Workshop on Local and Metropolitan Area Networks. IEEE, 2015, pp. 1-6.

[11] Y. Zhu, Z. Zhang, Z. Marzi, C. Nelson, U. Madhow, B. Y. Zhao, and H. Zheng, "Demystifying 60GHz outdoor picocells," in Proceedings of the 20th annual international conference on Mobile computing and networking. ACM, 2014, pp. 5-16.

[12] S. Sur, V. Venkateswaran, X. Zhang, and P. Ramanathan, "60 GHz indoor networking through flexible beams: A link-level profiling," in ACM SIGMETRICS Performance Evaluation Review, vol. 43, no. 1. ACM, 2015, pp. 71-84.

[13] C. Cordeiro, D. Akhmetov, and M. Park, "IEEE 802.11ad: Introduction and performance evaluation of the first multi-Gbps WiFi technology," in Proceedings of the 2010 ACM international workshop on mmWave communications: from circuits to networks. ACM, 2010, pp. 3-8.

[14] T. Azzino, M. Drago, M. Polese, A. Zanella, and M. Zorzi, "X-TCP: a cross layer approach for TCP uplink flows in mmWave networks," in 2017 16th Annual Mediterranean Ad Hoc Networking Workshop (MedHoc-Net). IEEE, 2017, pp. 1-6.

[15] Y. Li, Z. Pi, and P. Nuggehalli, "Methods and apparatus for flexible beam communications in random access in system with large number of antennas," 2016, US Patent 9,380,582.

[16] Z. Akhtar, Y. S. Nam, J. Chen, R. Govindan, E. Katz-Bassett, S. Rao, J. Zhan, and H. Zhang, "Understanding video management planes," in Proceedings of the Internet Measurement Conference 2018. ACM, 2018, pp. 238-251.

[17] G. R. MacCartney and T. S. Rappaport, "A flexible millimeter-wave channel sounder with absolute timing," IEEE Journal on Selected Areas in Communications, vol. 35, no. 6, pp. 1402-1418, 2017.

[18] F. Barzegar, D. J. Barnickel Jr, G. Blandino, I. Gerszberg, P. S. Henry, and T. M. Willis III, "Remote distributed antenna system," Mar. 272018 , US Patent 9,930,668. 